\title{
The interdialytic weight gain: a simple marker of left ventricular hypertrophy in children on chronic haemodialysis
}

\author{
Michael Fischbach $^{1} \cdot$ Ariane Zaloszyc $^{1} \cdot$ Rukshana Shroff $^{2}$
}

Received: 19 January 2015 / Revised: 25 February 2015 / Accepted: 3 March 2015 /Published online: 24 March 2015

(C) IPNA 2015

\begin{abstract}
Despite multiple advances in haemodialysis (HD) technology over the years, the morbidity and mortality of HD patients remain unacceptably high. Cardiovascular disease is the most common cause of death, and left ventricular hypertrophy (LVH), seen in two-thirds of children on dialysis, is a significant contributor. The importance of volume control is increasingly recognized by nephrologists and now considered to be as important as urea kinetics, both in the day-to-day management and the long-term outcome of dialysis patients. The results published by Paglialonga et al. (10.1007/s00467014-3005-2) in this issue of Pediatric Nephrology clearly demonstrate that there is a significant correlation between interdialytic weight gain (IDWG) and LVH in oligoanuric children on chronic HD and that children with an IDWG of $>4 \%$ are at high risk of LVH. One common practice to achieve euvolaemia is to prescribe very high ultrafiltration rates. However, both volume overload and aggressive fluid removal can induce circulatory stress and multi-organ injury. In adults, ultrafiltration rates of $>1.24 \%$ body weight per hour, even if well tolerated, are associated with a significant increase in mortality. Nephrologists should be aware of the risk of a high ultrafiltration rate, especially if tolerance is obtained by a positive dialysate-to-plasma sodium gradient. Haemodiafiltration, which allows for higher ultrafiltration rates with greater intradialytic haemodynamic stability, or
\end{abstract}

Michael Fischbach

Michel.Fischbach@chru-strasbourg.fr

1 Children's Dialysis Unit, University Hospital Strasbourg, CHU Hautepierre, 67098 Strasbourg, France

2 Renal Unit, Great Ormond Street Hospital for Children NHS Foundation Trust, London, UK more frequent and longer dialysis sessions allow for safe and effective fluid removal.

Keywords Chronic haemodialysis $\cdot$ Interdialytic weight gain · Left ventricular hypertrophy · Ultrafiltration rate . Children

\section{Introduction}

The study by Paglialonga et al. [1] published in this issue of Pediatric Nephrology demonstrates the impact of interdialytic weight gain (IDWG) on left ventricular hypertrophy (LVH) in oligoanuric children undergoing chronic haemodialysis (HD). Assessment of the IDWG is simple to perform and, as demonstrated by these authors, provides important information on cardiovascular status. Indeed, the authors demonstrate a significant correlation between IDWG and the left ventricular mass index, with those children with an IDWG of $>4 \%$ being at high risk of LVH. LVH has been associated with cardiovascular mortality in multiple paediatric and adult studies [2-7]. Here, we discuss the recent paradigm shift in the management of chronic dialysis patients, with a greater focus on volume control rather than a 'urea-centric' approach focussing on $\mathrm{Kt} /$ Valone [8]. As there is a strong association between salt intake and IDWG, assessment of the dietary and dialysate sodium content is an important part of the overall fluid management of the patient.

\section{Assessing dry weight and IDWG in children on chronic dialysis}

Any comprehensive approach to the problem of volume overload in chronic dialysis patients includes an assessment of dry 
weight and, consequently, determination of the IDWG. While many definitions of 'dry weight' are used, it is essentially the lowest tolerated postdialysis weight (i.e. without symptomatic intradialytic hypotension) that allows the patient to maintain a normal interdialytic blood pressure, ideally without the use of antihypertensive medications [3, 6]. Despite technological advances in intradialytic relative plasma volume monitoring [9, $10]$ and bioimpedance analysis $[11,12]$, the assessment of dry weight in children remains challenging [6]. However, these new technical tools provide objective measures to corroborate the clinical assessment of dry weight by the dialysis team. They are particularly useful in the assessment of dry weight in malnourished children, in whom excess fluid can mask the underlying emaciated state.

The online intradialytic relative plasma volume curve is the net result of the plasma refilling rate compared to the ultrafiltration rate, and allows for early detection and prevention of intradialytic hypotension $[9,13]$. Patients who are fluid overloaded will show a flat relative plasma volume slope over the entire dialysis session. Conversely, patients with a steep downslope of the plasma volume curve are more likely to be near their dry weight. If the curve shows a plateau after an initial drop [9], the plasma refilling rate has fully compensated for the ultrafiltration rate. In fact, the blood volume curve directly reflects one of the most important factors determining the plasma refilling rate, namely, the diffusive gradient of sodium between dialysate and plasma [13]. If a hypertonic dialysate sodium prescription is used (i.e. content of sodium in the dialysate sodium is higher than plasma sodium content), there is better preservation of intravascular volume due to an increased plasma refilling rate. A positive dialysate-to-plasma sodium gradient reduces the risk of intradialytic hypotension, but it can potentially leave the patient in a positive sodium balance, a risk factor for higher blood pressure and increased IDWG [13]. Conversely, a dialysate sodium concentration that is lower than the plasma sodium concentration is a risk factor for more frequent intradialytic hypotensive episodes, but it does reduce postdialytic thirst and, therefore, should limit the IDWG. Taken together, the on-line assessment of plasma volume allows for immediate detection and prevention of intradialytic hypotensive episodes, which cannot be achieved by a precise determination of the dry weight alone [9].

The bioimpedance body analysis is a reproducible and objective assessment of hydration and has been extensively studied in adults $[14,15]$ and children $[11,12]$ on dialysis. However, defining the dry weight from the total body water assessment, the most common parameter obtained from bioimpedance body analysis, can be difficult. The relationship between blood pressure and volume status is highly variable in adults [14] and children [12] on HD: extracellular volume assessment by bioimpedance analysis clearly demonstrates that blood pressure in the dialysis population is not always volume dependent (Fig. 1) [12, 14]. Systolic hypertension can be the result of extracellular overhydration, but also occurs in the case of extracellular underhydration. Conversely, hypotension is also not simply volume dependent $[12,14]$. Thus, physicians should be aware that a high blood pressure at the start of dialysis does not always imply extracellular overhydration and, therefore, cannot always be managed with increased ultrafiltration $[12,14]$. Lowering the extracellular volume to a normohydration or even underhydration status in order to optimize blood pressure control can be risky [3].

While the relation between a positive sodium balance and high systolic blood pressure is clearly related to extracellular volume expansion, which is turn is dependent on osmotically active sodium content, the concept of 'osmotically inactive sodium' with vasoactive properties was introduced by Titze [16]. Sodium can accumulate without water retention in the interstitium of the skin, leading to increased polymerization and sulfation of glycosaminoglycans in the sodium reservoir. This non-osmotic sodium seems to be of some importance in hypertension: subcutaneous tissue macrophages express the transcription factor tonicity enhancer binding protein in response to sodium-mediated interstitial osmotic stress and secrete vascular endothelial growth factor $\mathrm{C}$, which stimulates lymphatic formation and endothelial nitric oxide synthase expression, implying that the immune system is a regulator of volume and blood pressure homeostasis.

Taken together, salt plays a pivotal role in maintaining intravascular volume and, therefore, dialysis tolerance, but conversely it can lead to increased IDWG and hypertension. The accuracy of blood pressure measurement at dialysis start can be subject to many variables and measurement error, whereas IDWG is simple to assess and, as demonstrated by Paglialonga et al. [1], appears to be a more significant marker of LVH risk than blood pressure.

\section{Managing IDWG: safely achieving the desired ultrafiltration}

Interdialytic weight gain is correlated with the left ventricular mass index [1], a cardiovascular risk factor of morbidity and mortality $[2,5,7]$. Multiple interventions have been shown to be effective in reducing LVH and, consequently, in reducing cardiovascular mortality, with a recent special interest in extracellular volume control to achieve euvolaemia $[7,8,12,14$, 15]. To achieve euvolaemia many nephrologists prescribe high ultrafiltration rates [17], especially if the patient appears to tolerate it $[3,8]$. A patient's tolerance to a higher ultrafiltration rate depends on many factors, including maintaining normal haemoglobin levels, the use of bicarbonate dialysate buffer, higher dialysate sodium concentration [5], haemodiafiltration technology [18], ultrafiltration or sodium profiling, lowering dialysate temperature and on-line blood volume monitoring $[9,10]$, and has dramatically improved 
Fig. 1 Hydration measurement by bioimpedance spectroscopy and blood pressure $(B P)$ measurement in children on haemodialysis shows that only $31 \%$ of hypertensive children are overhydrated $(\mathrm{OH})$ (from Zaloszyc A et al. [12], used with permission)

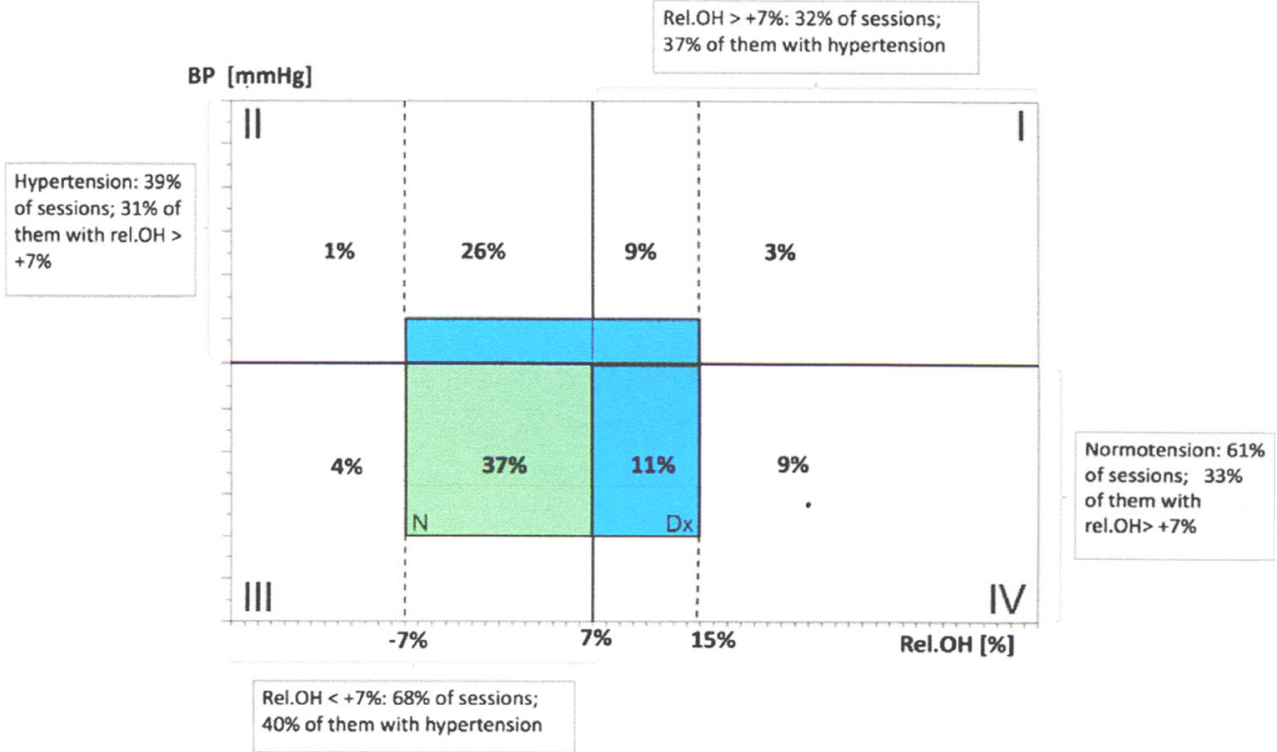

over the last decades [6]. Nonetheless, even a well-tolerated high ultrafiltration rate, such as $>1.24 \%$ body weight loss per hour in adults, is associated with greater mortality [19]. Thus, although a relationship between IDWG and left ventricular mass index is shown by Paglialonga et al. [1], the dialysis prescription used to reach the target dry weight should consider the risk of a high ultrafiltration rate, especially if tolerance is induced by a positive dialysate-to-plasma sodium gradient [3, 8, 13]. Clinical practice guidelines based on expert opinion have proposed limiting the ultrafiltration rate to $1.5 \%$ of body weight loss per hour in children [6], but in the light of recent studies, simply targeting a dry weight at the expense of high ultrafiltration rates is also harmful.

Where possible, more frequent and longer dialysis sessions $[20,21]$ should be considered, but for patients on in-centre three times/week dialysis, haemodiafiltration (HDF) provides a safe, well-tolerated and effective means of fluid removal. HDF is the addition of a determined convective volume to HD [18], thereby allowing for blood purification combining diffusive mass transport of small uraemic toxins and convective mass transfer of larger middle-molecular weight uraemic toxins. The total convective volume achieved over a HDF session is the sum of the prescribed weight loss and the predetermined ultrafiltration flow of blood, with ultrafiltration replaced by an equal volume of substitution fluid in order to achieve a large convective volume. The convective volume over and above the desired weight loss is replaced millilitre for millilitre with an intravenous infusion of substitution fluid produced 'on-line' from the dialysate. In on-line HDF dialysis, water is filtered through a reverse osmosis system followed by filtration through two disposable membranes in the dialysate circuit to produce 'ultra-pure' dialysis fluid (both 'ultra-pure' dialysate and 'ultra-pure' substitution fluid) with $>100$-fold lower bacterial and endotoxin levels than the water used for conventional HD.

A randomized controlled trial has shown that compared with conventional HD, convective therapies (HDF and haemofiltration) reduce symptomatic intradialytic hypotension in adults on long-term dialysis and that patients with cardiovascular instability during conventional HD treatments experience fewer side effects on HDF [22]. Several mechanisms may account for this phenomenon: differences in convective and diffusive sodium removal, different vascular reactivity or simply cooling of the blood. The core temperature of many dialysis patients is lower than normal, and an 'inappropriately' high dialysate temperature (e.g. $37^{\circ} \mathrm{C}$ ) may cause a transfer of heat from dialysate to the blood. On-line HDF may result in cooling of the blood via enhanced thermal energy losses within the extracorporeal system and may contribute to a significant reduction in the number of hypotensive episodes compared with HD. Today, modern HDF technology provides a tool for blood thermic monitoring that enables the nephrologist to achieve either normothermic balance, heating or cooling of the patient on HDF.

Finally, it must be kept in mind that antihypertensive medications may cause vasodilation and impede fluid removal from patients on HD despite volume overload [23]. Consequently, their use should be avoided where possible.

\section{IDWG: the role of dialysate sodium}

The IDWG is assumed to be an extracellular overhydration if body weight at dialysis start is above the prescribed dry weight. This presumed extracellular overhydration at dialysis start is often, but not always, associated with a high blood 
pressure [12, 14], and rarely with oedema. Nevertheless, it should be kept in mind that blood pressure and hydration do not correlate in approximately $40 \%$ of hydration assessments by body bioimpedance analysis (Fig. 1) [12, 14].

Since IDWG results from a gain of water and of sodium, it cannot simply be solved by isonatraemic ultrafiltration. Reducing IDWG needs more than simply a 'weight loss prescription' of ultrafiltration in millilitres, but a precise determination of the sodium balance that is influenced by a combination of convective sodium removal (i.e. a filtration process) and diffusive dialysis sodium removal (i.e. a diffusive gradient transfer). The dialysate sodium prescription should be adjusted to determine the dialysate-to-plasma sodium diffusive gradient $[3,13]$. Besides restricting interdialytic dietary salt intake, restricting dialysate sodium can reduce thirst, limit IDWG, assist in the achievement of dry weight and optimize blood pressure control [23]. It should also be noted that both volume overload and aggressive fluid removal can induce circulatory stress and multiorgan injury: euvolaemia achieved by rapid ultrafiltration can be potentially dangerous, even though it may appear to be well tolerated $[4,17,24]$.

\section{IDWG: nutrition and protein wasting}

Interdialytic weight gain may be the result of interdialytic food intake, good nutrition being essential for growth and to balance dialysis protein wasting [25]. In a thrice-weekly HD regimen, a higher IDWG is often managed by increasing the duration of the dialysis session or by prescribing additional time with isolated ultrafiltration $[6,7]$. In order to escape the consequences of longer dialysis, some children may stop eating so as to stay within the limits of their 'authorized' weight gain. This situation can lead to malnutrition and eventually to protein wasting $[6,21,25]$. Therefore, we believe in the concept of 'floating dry weight' [21], which is easy to apply with daily dialysis: the ultrafiltration rate is fixed at a safe percentage of body weight loss per hour [19, 20, 24], provided there are no clinical signs of overhydration.

Even if the prescribed dry weight is not achieved in a given session, it will be achieved before the end of a week of daily dialysis. Indeed, the dry weight is easily achieved over the whole dialysis week, applying daily ultrafiltration within safe limits of body weight loss per hour [6, 19-21, 26].

\section{Conclusion}

An IDWG of $>4 \%$ appears to be a simple and accurate predictor of LVH and is superior to blood pressure assessment alone in children on a thrice-weekly HD regimen [1]. On-line plasma volume monitoring and bioimpedance analysis can provide objective measures to corroborate the clinical assessment of dry weight and appropriate interpretation of blood pressure [12, 14, 27]. The dialysis prescription should be carefully individualized so that high ultrafiltration rates are avoided. In particular, the dialysate sodium content would need to be adjusted relative to the child's plasma sodium level so that postdialysis thirst is minimized, thereby limiting the increase in IDWG $[3,8,13]$. Importantly, in children on chronic HD, the IDWG reflects not only water and sodium intake, but also nutritional intake that is essential for growth [25], and dietary intake should not be reduced in an attempt to achieve a safe ultrafiltration rate $[4,6,17,19,20]$. In practice, the safest and most efficient dialysis prescription $[26,27]$ is intensive daily dialysis, with HDF offering safer and better tolerated ultrafiltration.

\section{References}

1. Paglialonga F, Consola S, Galli MA, Testa S, Edefonti A (2015) Interdialytic weight gain in oligo anuric children and adolescents on chronic haemodialysis. Pediatr Nephrol. doi:10.1007/s00467-0143005-2

2. Folley RN, Parfry PS, Sarnak MJ (1998) Clinical epidemiology of cardiovascular disease in chronic renal disease. Am J Kidney Dis 32:S112-S119

3. Agarwal R, Bouldin JM, Light RP, Garg A (2011) Probing dryweight improves left ventricular mass index. Am J Nephrol 33: 373-380

4. Hothi DK, Rees L, Marck J, Burton J, McIntyre CW (2009) Pediatric myocardial stunning underscortes the cardiac toxicity of conventional hemodialysis treatments. Clin J ASN 4:790-797

5. Oh J, Wunsch R, Turzer M, Bahner M, Raggi P, Querfeld U, Mehls O, Schaefer F (2002) Advanced coronary and carotid arteriopathy in young adults with childhood-onset chronic renal failure. Circulation 106:100-105

6. Fischbach M, Edefonti A, Schröder C, Watson A (2005) Hemodialysis in children: general practical guidelines. Pediatr Nephrol 20:1054-1066

7. Ulinski T, Genty J, Viau C, Tellous-Borde I, Deschenes G (2006) Reduction of left ventricular hypertrophy in children undergoing hemodialysis. Pediatr Nephrol 21:1171-1178

8. Davenport A (2011) How best to improve survival in hemodialysis patients: solute clearance or volume control? Kidney Int 80:10181020

9. Dheu C, Terzic J, Menouer S, Fischbach M (2009) Importance of the curve shape for interpretation of blood volume monitor changes during haemodiafiltration. Pediatr Nephrol 24:1419-1423

10. Patel HP, Goldstein SL, Mahan JD, Smith B, Fried CB, Currier H, Flynn JT (2007) A standard noninvasive monitoring of hematrocrit algorithm improves blood pressure control in pediatric hemodialysis patients. Clin J Am Soc Nephrol 2:252-257

11. Paglialonga F, Ardissino G, Galli MA, Scarfia RV, Testa S, Edefonti A (2012) Bioimpedance analysis and cardiovascular status in pediatric patients on chronic hemodialysis. Hemodial Int 16:S20-S25

12. Zaloszyc A, Schaefer B, Schaefer F, Krid S, Salomon R, Niaudet P, Schmitt CP, Fischbach M (2013) Hydration measurement by bioimpedance spectroscopy and blood pressure management in children on hemodialysis. Pediatr Nephrol 28:2169-2177

13. Santos FS, Peixoto AJ (2008) Revisiting the dialysate sodium prescription as a tool for better blood pressure and intradialytic weight 
gain management in hemodialysis patients. Clin J Am Soc Nephrol 3:522-530

14. Wabel P, Maissl U, Chamney P, Jirka T, Machek P, Ponce P, Taborsky P, Tetta C, Velasco N, Vlasak J, Zaluska W, Wizemann V (2008) Towards improved cardiovascular management: the necessity of combining blood pressure and fluid overload. Nephrol Dial Transplant 23:2965-2971

15. Chazot C, Wabel P, Chamney P, Moissl U, Wieskotten S, Wizemann V (2012) Importance of normohydration for the longterm survival of haemodiasis patients. Nephrol Dial Transplant 27: 2404-2410

16. Titze J, Luft FC, Buer K, Dietsch V, Lang R, Veelken R, Wagner H, Eckardt KU, Hilgers KF (2006) Extrarenal $\mathrm{Na}^{+}$balance, volume, and blood pressure homeostasis in intact and ovariectomized deoxycorticosterone acetate salt rats. Hypertension 47:1101-1107

17. Flyth JF, Brunelli SM (2011) The risk of high ultrafiltration rate in chronic hemodialysis: implications for patient care. Semin Dial 24: 259-265

18. Fischbach M, Fothergill H, Zaloszyc A, Seuge L (2012) Hemodiafiltration: the addition of a convective flow to hemodialysis. Pediatr Nephrol 27:351-356

19. Movilli E, Gaggia P, Zubani R, Camerini C, Vizzardi V, Parrinello G, Savoldi S, Fischer MS, Londrino F, Cancarini G (2007) Association between high ultrafiltration rates and mortality in uraemic patients on regular haemodialysis. A 5-year prospective observational multicentre study. Nephrol Dial Transplant 22: $3547-3552$
20. Locatelli F, Canaud B (2012) Dialysis adequacy today; a European perspective: morbidity, mortality, cardiovascular outcome, nutrition. Nephrol Dial Transplant 27:3043-3048

21. Fischbach M, Fothergill H, Zaloszyc A, Menouer S, Terzic J (2011) Intensified daily dialysis: the best chronic dialysis option for children? Semin Dial 24:640-644

22. Locatelli F, Altieri P, Andrulli S, Bolasco P, Sau G, Pedrini LA, Basile C, David S, Feriani M, Montagna G, Di Iorio BR, Memoli B, Cravero R, Battaglia G, Zoccali C (2010) Hemofiltration and hemodiafiltration reduce intradialytic hypotension in ESRD. J Am Soc Nephrol 21:1798-1807

23. Argawal R, Wier MR (2010) Dry weight: a concept revisited in an effort to avoid medication-directed approaches for blood pressure control in hemodialysis. Clin J Am Soc Nephrol 5:1255-1260

24. Rajan VR, Mitch E (2009) Muscle wasting in chronic kidney disease: the role of the ubiquitine proteasome system and its clinical impact. Pediatr Nephrol 23:527-535

25. Huang SH, Filler G, Lindsay R, McIntyre CW (2014) Euvolemia in hemodialysis patients: a potentially dangerous goal? Semin Dial. doi:10.1111/sdi 12317

26. Fischbach M, Terzic J, Menouer S, Dheu C, Seuge L, Zaloszyc A (2010) Daily on line haemodiafiltration promotes catch-up growth in children on chronic dialysis. Nephrol Dial Transplant 25:867873

27. Fischbach M, Zaloszyc A, Higel L, Menouer S, Terzic J (2014) Why does three times per week hemodialysis provide inadequate dialysis for children? Hemodial Int 18:S39-S42 